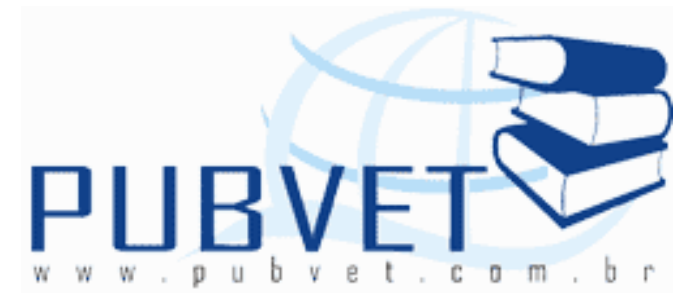

PUBVET, Publicações em Medicina Veterinária e Zootecnia.

\title{
Os caminhos na natureza da amônia oriunda da produção animal: Parte I - fontes, transformações e sumidouros
}

${\text { Luciano Barreto } \text { Mendes }^{(1)} \text {, Jofran Luiz de Oliveira }}^{(2)}$, Ilda de Fátima Ferreira Tinôco( ${ }^{(3)}$, Nico W. M. Ogink ${ }^{(4)}$

${ }^{(1)}$ Doutorando em Construções Rurais e Ambiência, Departamento de Engenharia Agrícola, Universidade Federal de Viçosa, Viçosa, Minas Gerais, Brasil. E-mail: mrmendes2010@gmail.com;

(2)Professor Assistente, Departamento de Engenharia Agrícola e Ambiental, Universidade Federal do Mato Grosso, Rondonópolis. E-mail: jofran@ufmt.br; ${ }^{(3)}$ Professora Adjunta, Departamento de Engenharia Agrícola, Universidade Federal de Viçosa, Viçosa. E-mail: iftinoco@ufv.br;

${ }^{(4)}$ Pesquisador Senior, Livestock Research Center, Wageningen University Research, Wageningen, Holanda. E-mail:nico.ogink@wur.nl.

\section{Resumo}

O fato de que uma vez lançada na atmosfera a amônia $\left(\mathrm{NH}_{3}\right)$ possui efeitos detrimentais nos ecossistemas circundantes, há muito deixou de ser uma mera hipótese para se tornar um paradigma científico. Isto combinado com o fato de que o setor da produção animal é o responsável pela emissão de 70 a $90 \%$ de toda a $\mathrm{NH}_{3}$ antropogênica lançada na atmosfera, torna a atividade agropecuária uma importante causa dos desequilíbrios ambientais advindos da dispersão deste gás. Após liberada na atmosfera, os processos envolvidos na 
MENDES, L.B. et al. Os caminhos na natureza da amônia oriunda da produção animal: Parte I fontes, transformações e sumidouros. PUBVET, Londrina, V. 6, N. 24, Ed. 211, Art. 1406, 2012.

dispersão e transformação da $\mathrm{NH}_{3}$ são complexos por natureza e de difícil observação experimental dada a escala em que ocorrem. No entanto tais processos precisam ser desnudados para a implementação de modelos mais realísticos do ciclo deste gás na natureza. Esta revisão de literatura foi elaborada com os seguintes objetivos: (1) expor a intrínseca relação existente entre os compostos $\mathrm{NH}_{3}$ e seu parceiro químico (o amônio, $\mathrm{NH}_{4}^{+}$), com foco nos processos naturais que regem a formação e volatilização; (2) versar sobre os processos de deposição da $\mathrm{NH}_{3}$ na natureza; e (3) apontar os potenciais sumidouros deste composto nitrogenado nos ecossistemas.

Palavras-chave: amônio, dispersão atmosférica, deposição da amônia.

\title{
The fate in nature of the ammonia originated from confined animal operations: Part I: sources, transformations and sinks
}

\begin{abstract}
The fact that once in the atmosphere, ammonia $\left(\mathrm{NH}_{3}\right)$ will have detrimental effects on the surroundings ecosystems has long left the category of hypothesis to become a scientific paradigm. This combined with what data have been suggesting that the animal production sector is responsible for emissions of 70 to $90 \%$ of all antropogenic $\mathrm{NH}_{3}$ released into the atmosphere, cause intensive animal operation systems to negatively impact the environment due to their gaseous emissions. Once released into the atmosphere, the processes involving $\mathrm{NH}_{3}$ dispersion and transformation are inherently complex and of difficult experimental observation due to the magnitude of its occurrence. However, such processes have to be denuded so that more accurate models for the $\mathrm{NH}_{3}$ cycle in nature can be implemented. This literature review was performed with the following objectives: (1) to show how $\mathrm{NH}_{3}$ and its chemical partner ammonium $\left(\mathrm{NH}_{4}^{+}\right)$are intrinsically related, focusing on the process that govern their formation, transformation and $\mathrm{NH}_{3}$ volatilization; (2) to depict the processes of deposition of $\mathrm{NH}_{3}$ in
\end{abstract}


MENDES, L.B. et al. Os caminhos na natureza da amônia oriunda da produção animal: Parte I fontes, transformações e sumidouros. PUBVET, Londrina, V. 6, N. 24, Ed. 211, Art. 1406, 2012.

nature; and (3) to indicate the potential sinks of this nitrogen compound in the ecosystems.

Keywords: ammonium, atmospheric dispersion, ammonia deposition

\section{Introdução}

As principais fontes de proteínas para a dieta humana possuem origem animal, sendo as carnes bovina, suína e de aves as mais comercializadas atualmente. Em relação à produção de carnes no mundo, observa-se que nas últimas duas décadas houve um incremento na produção de carne de frango, que respondia por $21 \%$ do total de carne produzida em 1990, e em 2010 representa $33 \%$ deste montante. A produção de carne suína manteve-se praticamente estável durante o período analisado, enquanto a produção de carne bovina apresentou decréscimo de $11 \%$ entre 1990 e 2010 . Esta mudança se dá em um perído de grandes alterações econômicas mundiais, onde a carne de aves se coloca no mercado como uma alternativa economicamente razoável (Figura 1). Outros fatores que justificam a alteração na matriz de produção de carnes no mundo é o custo das rações animais, e o fato de que dentre os três grupos de animais, as aves são as que possuem menor índice de conversão alimentar (CA), ou seja a relação entre consumo de ração e ganho de peso é menor. (United States Department of Agriculture USDA, 2010).



1990

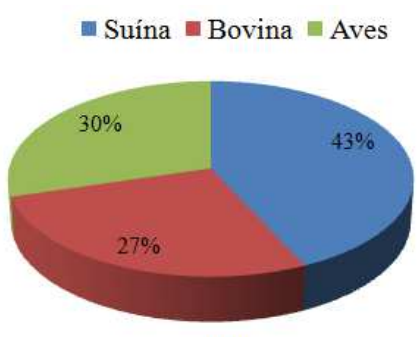

2000

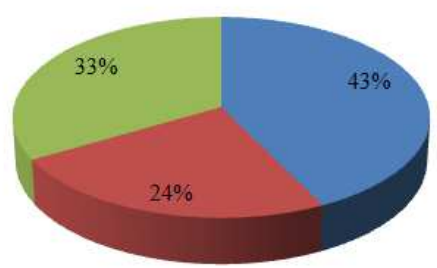

2010

Figura 1. Mudança temporal na composição da produção mundial dos principais tipos de carne. (Fonte: USDA, 2010) 
MENDES, L.B. et al. Os caminhos na natureza da amônia oriunda da produção animal: Parte I fontes, transformações e sumidouros. PUBVET, Londrina, V. 6, N. 24, Ed. 211, Art. 1406, 2012.

De acordo com o United Stated Department of Agriculture (USDA), em 2010, o Brasil ocupou o ranque de terceiro maior produtor mundial de carne de frango, produzindo $12.312 \mathrm{kt}$ de carcaça pronta para o consumo (16\% do total mundial), seguindo apenas os Estados Unidos da América (E.U.A.), produzindo 16.573 kt de carcaça; e a China, produzindo 12.550 kt de carcaça, ambos representando $22 \%$ e $16 \%$ do total mundial, respectivamente.

Tais posições no ranque mundial de produção de carne ocorrem graças ao advento dos sistemas intensivos de produção animal (SIPAs), aliados ao uso de dietas ricas em nutrientes no intuito de aumentar o desempenho zootécnico dos animais, as quais resultam na deposição de dejetos que são igualmente ricos em proteínas e outros compostos nitrogenados. Por exemplo, de acordo com Moro et al. (2005) a conversão alimentar (CA) de frangos de corte é de aproximadamente 1,97 , ou seja, praticamente $50 \%$ de toda a ração oferecida às aves é transformada em esterco.

Esterco e a cama usada de animais domésticos são as fontes principais das emissões de gases associadas aos sistemas intensivos de produção animal (SIPAs), dentre os quais pode-se citar a amônia $\left(\mathrm{NH}_{3}\right)$, como sendo um gás de grande importância. Estudos realizados na Europa no decorrer da última década têm reportado que cerca de 70 a $90 \%$ de toda a $\mathrm{NH}_{3}$ antropogênica emitida para atmosfera foi advinda dos SIPAs (Liu et al., 2006; Hutchings et al., 2001; USEPA, 2000; Pain et al., 1998; Battye et al.,1994).

A magnitude das emissões de $\mathrm{NH}_{3}$ para a atmosfera e sua direta correlação com os SIPAs têm preocupado a comunidade científica e os órgãos reguladores das emissões de poluentes para a atmosfera em países como os Estados Unidos, Canadá e alguns países Europeus. Por exemplo, em 2002 o Conselho Nacional de Pesquisa (National Research Council - NRC) dos E.U.A., publicou um relatório intitulado "Emissões gasosas oriundas de sistemas intensivos de produção animal: Conhecimento atual, necessidades futuras" (Air emissions from animal feeding operations: Current knowledge, future needs), o qual apontou a emissão de $\mathrm{NH}_{3}$ dos SIPAs como um fato preocupante nas escalas global, nacional e regional, sugerindo e estimulando a comunidade 
MENDES, L.B. et al. Os caminhos na natureza da amônia oriunda da produção animal: Parte I fontes, transformações e sumidouros. PUBVET, Londrina, V. 6, N. 24, Ed. 211, Art. 1406, 2012.

científica de então a intensificar os estudos sobre a emissão deste gás, como subproduto da indústria de criação de animais.

Por outro lado, a preocupação dos efeitos da $\mathrm{NH}_{3}$ originada dos SIPAs no meio-ambiente data do início dos anos 80, na Europa. Durante esta década, monitoramentos intensos das emissões de $\mathrm{NH}_{3}$ dos SIPAs em diversos países europeus foram realizados, e já em 1990, a "Directive on National Emission Ceilings for Certain Atmospheric Pollutants (NEC Directive)" determinou que até 2010 os níveis de emissão de $\mathrm{NH}_{3}$ dos SIPAs na União Européia deveriam ter sido reduzidos em 20\% dos níveis de referência medidos até 1990 (Grimm, 2009).

Várias têm sido as tentativas de se estimar o total global da emissão de $\mathrm{NH}_{3}$, como exposto nos trabalhos de Bowman et al. (1997) e Dentener e Crutzen (1994). O principal limitante para a melhora dos modelos de emissão é a falta de uma base de dados confiável e extensa que descreva o maior número possível de fontes emissoras e seus respectivos fatores de emissão, afim de que sirvam para melhorar os inventários e, a partir dos quais seja possível originar modelos mais confiáveis.

Do exposto, nota-se que a comunidade científica internacional tem despertado interesse e pesquisado incessantemente por mais de 30 anos, acerca da emissão de $\mathrm{NH}_{3}$ oriunda dos SIPAs, principalmente por seus impactos nos ecossistemas. O fato de que uma vez lançada na atmosfera, a $\mathrm{NH}_{3}$ possui efeitos detrimentais no meio-ambiente há muito tempo deixou de ser uma mera hipótese a ser testada para se tornar num paradigma científico (Sutton et al., 2008).

Todavia, mesmo com a magnitude da produção animal brasileira, tal preocupação dos efeitos ambientais da $\mathrm{NH}_{3}$ parece não existir para a comunidade científica e de legisladores. Como conseqüências disto, não existem metodologias adaptadas às condições brasileiras, ou bases de dados de emissão consistentes, nem tampouco políticas reguladoras relacionadas à emissão deste poluente. 
MENDES, L.B. et al. Os caminhos na natureza da amônia oriunda da produção animal: Parte I fontes, transformações e sumidouros. PUBVET, Londrina, V. 6, N. 24, Ed. 211, Art. 1406, 2012.

Faz-se válido mencionar que alguns esforços têm surgido a nível nacional no sentido de se monitorar ou estimar as concentrações de $\mathrm{NH}_{3}$ no interior de galpões avícolas, como os trabalhos publicados por Owada et al. (2007), Nääs et al. (2007), Medeiros et al. (2008) e Vigoderis et al. (2010), os quais tiveram como foco principal a qualidade do ar ao nível dos animais e trabalhadores. Em contrapartida, Saraz (2010) adaptou e testou uma cápsula de fluxo passivo (CFP) para a aferição de fluxos de $\mathrm{NH}_{3}$ da cama de aves; tal metodologia mostrou-se eficiente na estimativa dos fluxos, porém não leva em consideração a taxa de ventilação dos galpões monitorados, tornando o cálculo da taxa de emissão de $\mathrm{NH}_{3}$ uma tarefa difícil, sobretudo para galpões ventilados com pressão positiva, em vista da estimativa da ventilação ser mais complexa do que para sistemas ventilados sob pressão negativa (Baptista et al, 1999). O uso de cápsulas de fluxo passivo para estimar fatores de emissão de $\mathrm{NH}_{3}$ pode ser uma tarefa complexa por que a volatilização deste gás é extremamente dependente da ventilação (Ogink et al. 2012). Souza e Melo (2011) conduziram um inventário da emissão de $\mathrm{NH}_{3}$ oriundo do setor de criação de animais domésticos para o estado do Rio de Janeiro, sendo que os fatores de emissão utilizados foram aqueles estimados por Bowman et al (1997), os quais por sua vez foram calculados a partir dos fatores determinados experimentalmente para países desenvolvidos e multiplicados por índices de redução baseados na pressuposição de que em países em desenvolvimento o total de animais confinados ou enclausurados é menor do que em países desenvolvidos.

Estudos na antiga Alemanha Ocidental estimaram emissão total de amônia a partir de instalações de produção animal de até 700000 t/a. Da quantidade emitida, calcula-se que entre 10 e $23 \mathrm{~kg} / \mathrm{ha}$ de nitrogênio por ano penetra no solo, sendo esta média até mesmo superior à carga crítica para a maioria dos habitats naturais. A amônia tem efeito direto sobre as plantas nas áreas circundantes, e também é transportada por longas distâncias através do ar, causando a eutrofização e acidificação da água e solo, resultando em alterações na ecologia vegetal (Hartung e Phillips, 1994). 
MENDES, L.B. et al. Os caminhos na natureza da amônia oriunda da produção animal: Parte I fontes, transformações e sumidouros. PUBVET, Londrina, V. 6, N. 24, Ed. 211, Art. 1406, 2012.

É diante da perplexidade advinda deste cenário preocupante que se conduziu a presente revisão bibliográfica, a qual tem como principais objetivos os seguintes: (1) expor a intrínseca relação existente entre os compostos amônia e amônio, com foco nos processos naturais que regem a formação e volatilização da amônia a partir do amônio; (2) versar sobre os processos de deposição da amônia na natureza; e (3) apontar os potenciais sumidouros deste composto nitrogenado nos ecossistemas.

\section{Amônia versus amônio: um elétron que faz a diferença}

A $\mathrm{NH}_{3}$ nas condições normais de temperatura $\left(25^{\circ} \mathrm{C}\right)$ e pressão (1 ATM) (STP) encontra-se no estado gasoso, o qual é incolor e possui odor pungente (Slack e James, 1973). A molécula de amônia é formada por um átomo de nitrogênio e três de hidrogênio cuja fórmula molecular é $\mathrm{N}-\mathrm{H}_{3}$. O átomo de nitrogênio central possui cinco valências ou elétrons livres, três dos quais se ligam com os respectivos elétrons livres dos três átomos de hidrogênio, restando um par de elétrons. A geometria da molécula, portanto, possui formato piramidal-trigonal (Figura 2). No caso da molécula de $\mathrm{NH}_{3}$, os ângulos entre as ligações $\mathrm{H}-\mathrm{N}-\mathrm{H}$ são de $107,8^{\circ}$, um pouco menor que a ligação existente entre ligações $\mathrm{H}-\mathrm{O}-\mathrm{H}$ na molécula de água que é de 109,470. Este desvio é resultado da repulsão existente entre o par de elétrons não ligantes e os pares de elétrons das ligações $\mathrm{N}-\mathrm{H}$. O fato de o átomo de nitrogênio na molécula de $\mathrm{NH}_{3}$ possuir um par de elétrons livres torna a amônia uma base, ou um receptor de prótons (Lee, 2009).

$\mathrm{O}$ formato da molécula de $\mathrm{NH}_{3}$ confere à mesma um momento dipolar resultante diferente de zero, ou seja, o centro de carga negativa não coincide com o centro de carga positiva, tornando-a polar. A polaridade da molécula de $\mathrm{NH}_{3}$ e sua habilidade em formar ligações de hidrogênio tornam-na uma substância altamente miscível em água. $\mathrm{A} \mathrm{NH}_{3}$ possui $\mathrm{pH}$ moderadamente básico, sendo que $1,0 \mathrm{~mol}$ de solução aquosa possui um $\mathrm{pH}$ de 11,6 e se um ácido forte é adicionado a tal solução até a neutralização total da mesma ( $\mathrm{pH}$ 
MENDES, L.B. et al. Os caminhos na natureza da amônia oriunda da produção animal: Parte I fontes, transformações e sumidouros. PUBVET, Londrina, V. 6, N. 24, Ed. 211, Art. 1406, 2012.

$=7), 99,4 \%$ da $\mathrm{NH}_{3}$ será protonada. Algumas propriedades da molécula de $\mathrm{NH}_{3}$ podem ser observadas na Tabela 1.

Tabela 1. Algumas propriedades físicas e químicas da amônia

\begin{tabular}{cc}
\hline Massa molar & $17.031 \mathrm{~g} \mathrm{~mol}^{-1}$ \\
Densidade & $0.73 \mathrm{~kg} \mathrm{~m}^{-3}\left(1.013 \mathrm{bar}\right.$ a $\left.15^{\circ} \mathrm{C}\right)$ \\
Ponto de fusão & $-77^{\circ} \mathrm{C}$ \\
Ponto de ebulição & $-33,4^{\circ} \mathrm{C}$ \\
Solubilidade em água & $31 \%\left(25^{\circ} \mathrm{C}\right)$ \\
Acidez & $9,3 \mathrm{pK}_{\mathrm{a}}$ (água $)$ \\
Basicidade & $4,75 \mathrm{pK}_{\mathrm{b}}$ \\
\hline
\end{tabular}

Fonte: Lee, 2009.

O íon amônio, também chamado de amônia ionizada, amínio ou azânio é uma estrutura poliatômica positivamente carregada sendo, portanto um cátion, cuja formula química é $\mathrm{NH}_{4}^{+}$.

De acordo com Lee (2009), uma base é uma espécie química que possui pelo menos um par de elétrons não-ligantes disponível para compartilhar com outra espécie durante uma reação química, enquanto que um ácido é uma espécie capaz de aceitar um par de elétrons. Portanto em uma reação química a $\mathrm{NH}_{3}$ comporta-se como base o íon $\mathrm{NH}_{4}{ }^{+}$como ácido.

Outra teoria geral de ácidos foi proposta por Brønsted-Lowri, segundo a qual, ácidos são definidos como substâncias capazes de doar prótons e base são substâncias capazes de aceitar. A base ao receber um próton é convertida no ácido conjugado (Barbosa, 2000). Portanto, em solução aquosa o íon $\mathrm{NH}_{4}{ }^{+}$ se comporta como ácido, cuja força é dada pela constante $K_{a}$ (Equação 1), denominada constante de ionização (Lee, 2009).

$$
k_{a}=\frac{\left[\mathrm{NH}_{3}\right]\left[\mathrm{H}_{3} \mathrm{O}^{+}\right]}{\left[\mathrm{NH}_{4}^{+}\right]}[1]
$$


MENDES, L.B. et al. Os caminhos na natureza da amônia oriunda da produção animal: Parte I fontes, transformações e sumidouros. PUBVET, Londrina, V. 6, N. 24, Ed. 211, Art. 1406, 2012.

Outra maneira de se representar a acidez é pelo valor de $\mathrm{pK}_{\mathrm{a}}$ que é definido como:

$$
p K_{a}=-\log \left[H^{+}\right][2]
$$

Quanto maior o valor de $\mathrm{K}_{\mathrm{a}}$, menor será o valor de $\mathrm{pK}_{\mathrm{a}}$ e mais forte será o ácido. Portanto, o pKa mede a tendência da molécula se ionizar. E esta tendência aumenta cerca de dez vezes quando o pH aumenta de uma unidade. $\mathrm{O} \mathrm{pK}_{\mathrm{a}}$ do $\mathrm{NH}_{4}{ }^{+}$é 9,24, o que o insere na categoria dos ácidos fracos. Assim com o aumento do $\mathrm{pH}$ da solução a tendência do íon $\mathrm{NH}_{4}{ }^{+}$se ionizar aumenta. Em valores abaixo do seu $\mathrm{pK}_{\mathrm{a}}$ a forma predominante é a espécie $\mathrm{NH}_{4}{ }^{+}$. No valor de $\mathrm{pH}$ próximo do $\mathrm{pK}_{\mathrm{a}} \mathrm{o}$ íon amônio começa a se ionizar. Quando se atinge o valor de $\mathrm{pH}$ correspondente ao $\mathrm{pKa}$, a solução terá $50 \%$ da forma protonada $\left(\mathrm{NH}_{4}{ }^{+}\right)$e $50 \%$ da forma desprotonada $\left(\mathrm{NH}_{3}\right)$. Em valores bem acima do pKa o íon $\mathrm{NH}_{4}{ }^{+}$já se ionizou e a forma predominante em solução é a espécie $\mathrm{NH}_{3}$ (Nelson e Cox, 2011).

Portanto, a intensidade na qual $\mathrm{NH}_{3}$ forma íons de $\mathrm{NH}_{4}^{+}$dependerá do $\mathrm{pH}$ da solução. Se o pH for baixo, o equilíbrio da reação química da Equação 3 é deslocado para a esquerda: mais moléculas de $\mathrm{NH}_{3}$ serão convertidas em íons de $\mathrm{NH}_{4}^{+}$. Se o pH for alto (a concentração de íons hidrônio é baixa), o equilíbrio da reação é deslocado para a direita: o íon hidróxido subtrai um próton do íon $\mathrm{NH}_{4}^{+}$, produzindo $\mathrm{NH}_{3}$ (Nelson e Cox, 2011).

$N H_{4}^{+}(l) \leftrightarrow N_{3}(l)+H_{3} O^{+}[3]$

\section{Relação entre amônia e o amônio presentes nos resíduos animais}

Boa parte da $\mathrm{NH}_{3}$ encontrada na natureza é essencialmente o produto da degradação de compostos orgânicos nitrogenados. Os processos bioquímicos de degradação do ácido úrico (Equação 4), uréia (Equação 5) e proteínas não- 
MENDES, L.B. et al. Os caminhos na natureza da amônia oriunda da produção animal: Parte I fontes, transformações e sumidouros. PUBVET, Londrina, V. 6, N. 24, Ed. 211, Art. 1406, 2012.

digeridas (Equação 6) são complexos, mas podem ser simplificados como a seguir (Groot Koerkamp et al., 1998):

$$
\mathrm{C}_{5} \mathrm{H}_{4} \mathrm{O}_{3} \mathrm{~N}_{4}+1,5 \mathrm{O}_{2}+4 \mathrm{H}_{2} \mathrm{O} \rightarrow 5 \mathrm{CO}_{2}+4 \mathrm{NN}_{3}
$$

$\mathrm{CO}\left(\mathrm{NH}_{2}\right)+\mathrm{H}_{2} \mathrm{O} \rightarrow \mathrm{CO}_{2}+2 \mathrm{NH}_{3} \quad$ [5]

Proteínas não-digeridas $\rightarrow \mathrm{NH}_{3}$ [6]

Uma vez formada na solução do esterco ou cama de animais pelo processo de desnitrificação (ou mineralização), a $\mathrm{NH}_{3}$ é passível de volatilização para o ar. Antes de ser liberada para o ar das vizinhanças, a $\mathrm{NH}_{3}$ entra em equilíbrio nas suas formas liquida e gasosa (Groot Koerkamp et al., 1998).

$\mathrm{O}$ equilíbrio entre $\mathrm{NH}_{3}$ e $\mathrm{NH}_{4}^{+}$é grandemente influenciado pela temperatura da solução e pelo $\mathrm{pH}$. Em valores de $\mathrm{pH}$ abaixo de 7, a $\mathrm{NH}_{3}$ da solução no esterco ou na cama tenderá a converte-se em $\mathrm{NH}_{4}{ }^{+}$, a qual não é passível de volatilização. Altas temperaturas favorecem a volatilização da $\mathrm{NH}_{3}$ graças à influência positiva da temperatura na constante de dissociação da reação (Equação 3).

$\mathrm{O}$ equilíbrio da volatilização da $\mathrm{NH}_{3}$ para a fase gasosa segue a lei de Henry para sistemas diluídos (Equações 7 e 8).

$$
\begin{aligned}
& \mathrm{NH}_{3}(l) \leftrightarrow \mathrm{NH}_{3}(\mathrm{~g})[7] \\
& N H_{3}(\mathrm{~g}, \text { esterco ou cama }) \leftrightarrow N H_{3}(g, \text { ar })[8]
\end{aligned}
$$

Para o caso específico da cama de frangos de corte e esterco de galinhas poedeiras, o melhor processo químico que descreve a geração de $\mathrm{NH}_{3}$ está representado na Equação 4, onde os íons $\mathrm{NH}_{4}{ }^{+}$são particionados entre as fases adsorvida e dissolvida (Figura 2). $\mathrm{NH}_{3}$ é transmitida da superfície da partícula de esterco para 0 ar do ambiente circundante através da transferência de massa por convecção. 
MENDES, L.B. et al. Os caminhos na natureza da amônia oriunda da produção animal: Parte I fontes, transformações e sumidouros. PUBVET, Londrina, V. 6, N. 24, Ed. 211, Art. 1406, 2012.

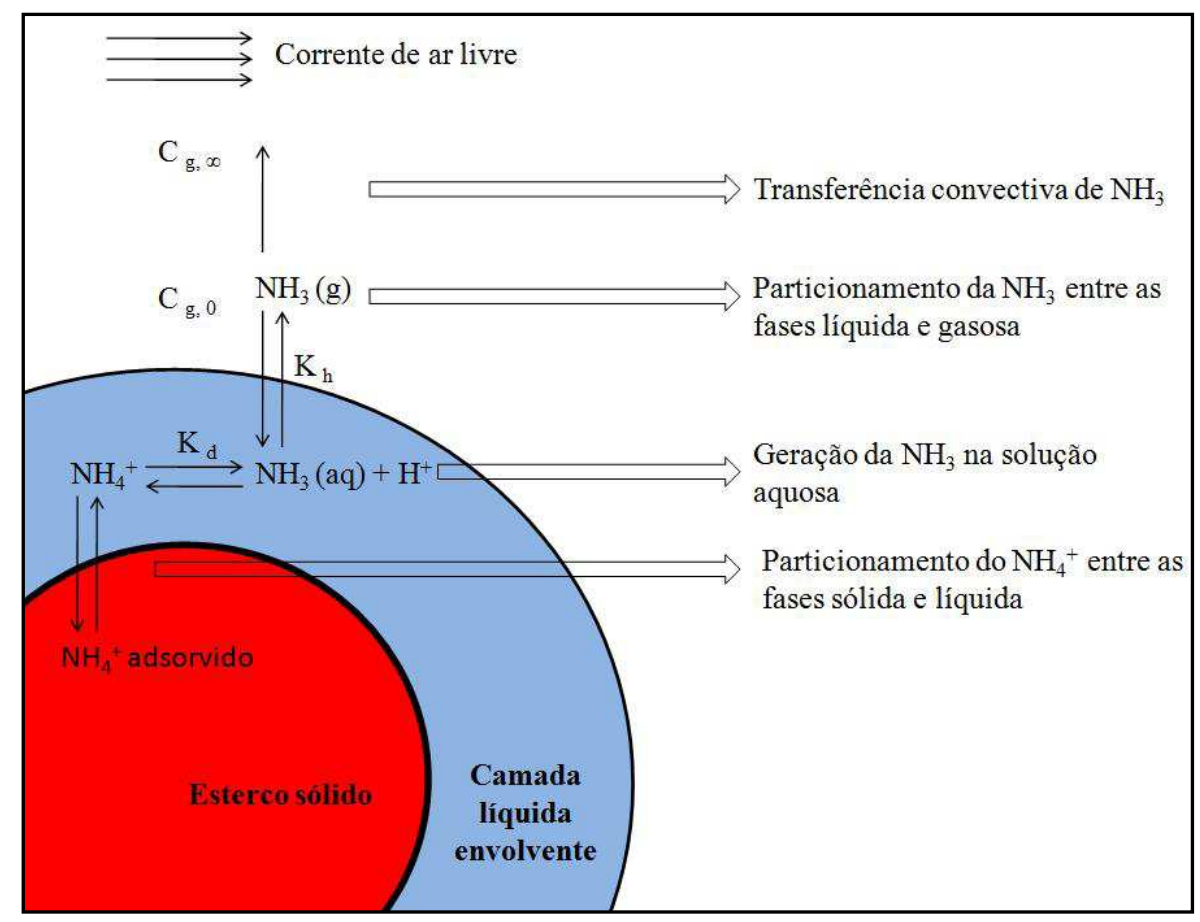

Figura 2. Ilustração dos processos relacionados com a emissão de $\mathrm{NH}_{3}$ do esterco sólido (Adaptado de Liu et al., 2006)

Estimativas apontam que o tempo de vida da $\mathrm{NH}_{3}$ na atmosfera está na faixa de 0,5 hora a 5 dias (Walker et al., 2001; Fowler et al., 1997). Este curto tempo de permanência é o resultado da rápida conversão da $\mathrm{NH}_{3}$ em $\mathrm{NH}_{4}{ }^{+} \mathrm{e}$ da deposição da $\mathrm{NH}_{3}$ em superfícies presentes na natureza, principalmente superfícies úmidas e vegetação. Uma vez na atmosfera, a $\mathrm{NH}_{3}$ que não é depositada a seco ou 'lavada' pelas gotas da chuva será transformada em aerosóis de $\mathrm{NH}_{4}{ }^{+}$. O tempo de vida dos aerosóis de $\mathrm{NH}_{4}{ }^{+}$é tipicamente de 5 a 10 dias (Walker et al., 2001; Crutzen, 1983). A taxa de conversão tem um impacto importante nos efeitos regionais da $\mathrm{NH}_{3}$ emitida sendo que a transformação da $\mathrm{NH}_{3}$ em $\mathrm{NH}_{4}{ }^{+}$depende de fatores como temperatura do ar, concentração de ácidos fortes e vapor de água na atmosfera. $\mathrm{A} \mathrm{NH}_{3}$ pode reagir com os ácidos sulfúrico, nítrico e clorídrico para formar sulfato de amônio, bi-sulfato de amônio, nitrato de amônio e cloreto de amônio. Os aerosóis de $\mathrm{NH}_{4}{ }^{+}$formados nestas reações podem existir como partículas 
MENDES, L.B. et al. Os caminhos na natureza da amônia oriunda da produção animal: Parte I fontes, transformações e sumidouros. PUBVET, Londrina, V. 6, N. 24, Ed. 211, Art. 1406, 2012.

sólidas ou gotículas de solução a depender da umidade relativa do ar (Walker et al., 2001; Finlayson-Pitts and Pitts, 1986).

A razão entre $\mathrm{NH}_{3}$ e $\mathrm{NH}_{\mathrm{x}}$ tem sido utilizada para identificar a fonte de $\mathrm{NH}_{\mathrm{x}}$ e a contribuição relativa da $\mathrm{NH}_{3}$ ou do $\mathrm{NH}_{4}{ }^{+}$para a deposição total de $\mathrm{NH}_{x}$ (Shen et al., 2011; Walker et al., 2004). Quando a razão é maior do que 0,5, significa que $\mathrm{NH}_{x}$ é composta basicamente de fontes locais de $\mathrm{NH}_{3}$, e a deposição a seco da $\mathrm{NH}_{3}$ é quem mais contribui para a deposição total de $\mathrm{NH}_{\mathbf{x}}$. Como $\mathrm{O} \mathrm{NH}_{4}{ }^{+}$é derivado da $\mathrm{NH}_{3}$ oriunda de fontes de emissão relativamente distantes, quando a razão de $\mathrm{NH}_{3}$ para $\mathrm{NH}_{\mathrm{x}}$ for menor do que 0,5 , significa que o total de $\mathrm{NH}_{\mathrm{x}}$ encontrado na atmosfera da área de estudo é afetada pelo $\mathrm{pNH}_{4}{ }^{+}$(aerosóis de $\mathrm{NH}_{4}{ }^{+}$) transportado por longas distâncias e a deposição de $\mathrm{NH}_{\mathrm{x}}$ é dominada pelas deposições úmida e a seco de $\mathrm{pNH}_{4}{ }^{+}$(Shen et al., 2011).

\section{Processos de deposição de $\mathrm{NH}_{x}$}

\subsection{Deposição a seco de $\mathrm{NH}_{3} \underline{\mathrm{NH}}_{4}{ }^{+}$}

Até o início do século $X X$, já era bem conhecido pela comunidade científica a existência de uma quantidade significativa de $\mathrm{NH}_{3}$ e $\mathrm{NH}_{4}{ }^{+}$no ar. Também era sabido que as plantas poderiam absorver $\mathrm{NH}_{3}$ diretamente da atmosfera, mas pouco se sabia se tais fluxos seriam importantes do ponto de vista prático. Os primeiros estudos que versam sobre esta temática surgiram no campo da micrometeorologia a respeito da deposição a seco da $\mathrm{NH}_{3}$ foram conduzidos por Hall e Miller (1911), cujos objetivos eram estimar a absorção direta da $\mathrm{NH}_{3}$ do ar por plantas com diferentes tipos de copa, o que serviria como uma contribuição adicional juntamente com $0 \mathrm{NH}_{4}{ }^{+}$recebido na precipitação para o total de $\mathrm{N}$ advindo da atmosfera (Sutton et al., 2008).

Define-se como deposição a seco (DS), todo tipo de deposição que ocorre sem a presença de água como meio de transporte. O orvalho sobre as plantas tem papel importante na DS por melhorar a aderência do material precipitado com a superfície foliar. Monitoramentos da DS são difíceis de realizar, e geralmente ocorrem de forma indireta (Moumen et al., 2004). A 
MENDES, L.B. et al. Os caminhos na natureza da amônia oriunda da produção animal: Parte I fontes, transformações e sumidouros. PUBVET, Londrina, V. 6, N. 24, Ed. 211, Art. 1406, 2012.

velocidade de deposição $\left(v_{d}\right)$ pode ser estimada pela soma das resistências representada na Equação 9 (Dentener e Crutzen, 1994).

$\frac{1}{v_{d}}=R_{a}+R_{b}+R_{c}$

Em que $v_{d}$ é medida em $\left(m^{-1}\right), R_{a}$ é a resistência aerodinâmica $\left(s^{-1}\right) d a$ superfície de deposição, $R_{b}$ é a resistência da sub-camada laminar $\left(s \mathrm{~m}^{-1}\right)$ entre a superfície e a camada limite turbulenta, e $R_{c}$ é a resistência da copa da vegetação $\left(\mathrm{s} \mathrm{m}^{-1}\right)$, neste caso considerada como sendo o recíproco da condutância estomatal das folhas.

Uma série de fatores pode influenciar a DS, indo desde condições meteorológicas e químicas da atmosfera até estrutura da copa, microestrutura das folhas e características biológicas, químicas e físicas da superfície receptora. O processo de DS usualmente ocorre com gases e partículas pequenas $(<2 \mu \mathrm{m})$, e pode ser dividido em três passos (Anderson e Hovman, 1999; Wesley e Hicks, 1977): (1) o transporte turbulento de matéria da atmosfera para as proximidades da copa; (2) a difusão do material através da camada limite superficial (espessura da camada de ar estagnado que envolve a superfície das plantas é geralmente $0,1-1 \mathrm{~mm}$ ); (3) adsorção pelas superfícies úmidas ou pegajosas e/ou captura pelos estômatos para o interior da planta.

Para partículas maiores que possuem sua massa influenciada pela gravidade, a DS ocorrerá através da sedimentação e colisão das partículas com a superfície das plantas ou do solo (Andersen e Hovman, 1999).

A taxa de DS estimada por modelos matemáticos é raramente validada com experimentos de laboratório ou de campo. Os métodos de medição mais utilizados são baseados em técnicas aplicadas a micrometeorologia, os quais requerem instrumentos sofisticados e onerosos. Outros métodos fazem uso da 
MENDES, L.B. et al. Os caminhos na natureza da amônia oriunda da produção animal: Parte I fontes, transformações e sumidouros. PUBVET, Londrina, V. 6, N. 24, Ed. 211, Art. 1406, 2012.

superfície natural dos vegetais e/ou o uso de superfície manufaturadas pelo homem, para estimar diretamente o fluxo da deposição de um poluente.

Apesar de todos os esforços da comunidade científica nos últimos 20 anos para medir DS, um método universalmente aceito para medir fluxos de DS parece ainda não existir (Moumen et al., 2004). A eficiência de uma coleção de materiais utilizados como superfícies de deposição (placas Petri, Teflon, vidro, plástico, papéis-filtro, etc) tem sido estudados durante as ultimas décadas (Tsitouridou, 2007).

Erisman et al. (1996) reportaram valores de $\mathrm{DS}$ de $\mathrm{NH}_{\mathrm{x}}$ para a floresta Speuld, na Holanda como sendo 17,9 e 4,7 kg ha- ano ${ }^{-1}$ de $\mathrm{NH}_{3} \mathrm{e} \mathrm{NH}_{4}{ }^{+}$, respectivamente. De acordo com Krupa (2003), plantas expostas a níveis elevados de $\mathrm{NH}_{3}$ apresentam injúrias visíveis nas folhas. Isto ocorre porque a taxa de absorção pelas folhas pode ser maior do que a taxa de desintoxicação 'in vivo' das mesmas.

\subsection{Deposição úmida de $\mathrm{NH}_{3}$ e NH$_{4}{ }^{+}$}

A deposição úmida (DU) ocorre durante as chuvas (precipitações), garoas ou chuvas de granizo, sendo água o meio de transporte das substâncias de interesse. As técnicas de monitoramento da DU são bem desenvolvidas, sendo diretamente estimada pela medição da concentração iônica do constituinte de interesse $\left(\mathrm{NH}_{3}\right.$ ou $\mathrm{NH}_{4}{ }^{+}$) na água de precipitação (Moumen et al., 2004).

Um exemplo de parametrização do processo de DU é o apresentado por Junge e Gustafson (1957), citados por Dentener e Crutzen (1994), representada pela Equação 10 .

$P=\frac{\varepsilon \times R}{L}[10]$

Em que $\mathrm{P}\left(\mathrm{s}^{-1}\right)$ é o coeficiente de remoção úmida; $\varepsilon$ (adimensional) representa a eficiência da diluição das partículas nas gotículas de água presentes na 
MENDES, L.B. et al. Os caminhos na natureza da amônia oriunda da produção animal: Parte I fontes, transformações e sumidouros. PUBVET, Londrina, V. 6, N. 24, Ed. 211, Art. 1406, 2012.

atmosfera; $\mathrm{L}\left(\mathrm{g} \mathrm{m}^{-3}\right.$ ) é o conteúdo de água no estado líquido na nuvem e R ( $\mathrm{g}$ $\mathrm{m}^{-3} \mathrm{~s}^{-1}$ ) é uma função da taxa de precipitação.

Os processos através dos quais gases e aerosóis são removidos pela precipitação podem ser divididos em duas classes: (a) processos que ocorrem dentro das nuvens e (b) processos que ocorrem abaixo do nível das nuvens. Os processos que ocorrem abaixo das nuvens incluem a remoção inercial e a remoção difusiva, ambas causadas pela precipitação. Os processos que ocorrem no interior das nuvens, e que resultam na DU de aerosóis e gases incluem a remoção inercial das partículas de nuvens, nucleação e difusão para as gotículas das nuvens (Walker et al., 2001; Twomey, 1977).

É consenso dentre pesquisadores especialistas que os processos que ocorrem no interior das nuvens são mais eficientes do que os que ocorrem abaixo do nível das nuvens. Uma razão para isto é o fato de que a área superficial total das gotículas de água no interior das nuvens é maior que na base da nuvem. A contribuição dos processos que ocorrem abaixo do nível das nuvens, porém, não podem ser ignorados especialmente nos casos em que a concentração do composto de interesse é maior abaixo das nuvens do que no interior das mesmas. Deve-se atentar para o fato de que certa quantidade do $\mathrm{NH}_{4}{ }^{+}$medido na precipitação é o resultado da DS dos aerosóis de $\mathrm{NH}_{4}{ }^{+}$no coletor de precipitação. É importante notar também que uma fração do $\mathrm{NH}_{4}{ }^{+}$ medido na água das chuvas é originada do gás $\mathrm{NH}_{3}$ presente na água graças aos processos de deposição seca ou úmida, quando a $\mathrm{NH}_{3}$ reage com água (Walker et al., 2001; Warneck, 1988).

\section{Sumidouros de $\mathrm{NH}_{3}$ e $\mathrm{NH}_{4}{ }^{+}$na natureza}

Uma parcela da $\mathrm{NH}_{3}$ presente na atmosfera que não se transformou em $\mathrm{NH}_{4}{ }^{+}$será adsorvida pelos vegetais, como já mencionado anteriormente (deposição a seco de $\mathrm{NH}_{3}$ ), através dos estômatos foliares. A outra parte da $\mathrm{NH}_{3}$ pode chegar à superfície através da deposição úmida, a qual no solo, a depender do $\mathrm{pH}$ do mesmo, pode (a) converter-se em $\mathrm{NH}_{4}{ }^{+}$, ou (b) então 
MENDES, L.B. et al. Os caminhos na natureza da amônia oriunda da produção animal: Parte I fontes, transformações e sumidouros. PUBVET, Londrina, V. 6, N. 24, Ed. 211, Art. 1406, 2012.

retornar à atmosfera através da volatilização, ou ainda (c) ser lixiviada para lençóis freáticos, alcançando corpos d'água.

Do total de $\mathrm{NH}_{4}{ }^{+}$formado na atmosfera, uma parte poderá ser depositada a seco ou através da precipitação para as superfícies, ao passo em que a outra parte pode reagir com gases presentes na atmosfera e formar aerosóis inorgânicos secundários, que são inertes. $\mathrm{NH}_{4}^{+}$e seus produtos podem permanecer na atmosfera por um tempo maior do que $\mathrm{NH}_{3}$, porém uma vez que atingem a superfície, poderão alcançar a solução do solo e os íons $\mathrm{NH}_{4}{ }^{+}$poderão (a) ser adsorvidos pelas plantas na forma de nitritos e/ou nitratos, ou (b) ser lixiviados para lençóis freáticos e alcançar corpos d'água superficiais.

Nos corpos d'água, $\mathrm{O} \mathrm{NH}_{4}{ }^{+}$oriundo da dissolução dos aerosóis ou da reação de protonação da $\mathrm{NH}_{3}$ poderá ser convertido em nitritos ou nitratos e posteriormente ser incorporado por organismos como as algas.

\section{Considerações Finais}

Amônia $\left(\mathrm{NH}_{3}\right)$ e amônio $\left(\mathrm{NH}_{4}^{+}\right)$presentes na natureza estão intrinsecamente correlacionados, de modo que em uma solução aquosa, $\mathrm{NH}_{3}$ é constantemente transformada em $\mathrm{NH}_{4}{ }^{+}$e vice-versa, sendo que a concentração dos dois depende de fatores como pH e temperatura da solução. Soluções aquosas ácidas conterão mais $\mathrm{NH}_{4}{ }^{+}$do que $\mathrm{NH}_{3}$, e o oposto será verdade quando a solução tender à basicidade. $\mathrm{NH}_{3}$ é altamente volatilizável enquanto que $\mathrm{NH}_{4}{ }^{+}$não é, e esta informação é muito importante ao desenvolverem-se técnicas de abatimento da emissão de $\mathrm{NH}_{3}$.

Depois de emitida para a atmosfera, a $\mathrm{NH}_{3}$ pode converter-se novamente em $\mathrm{NH}_{4}{ }^{+}$e potencialmente formar aerosóis. A relação entre $\mathrm{NH}_{3}$ e $\mathrm{NH}_{4}{ }^{+}$, ambos medidos na atmosfera, é um indicativo do tempo de permanência da $\mathrm{NH}_{3}$ na atmosfera, sendo que altas concentrações de $\mathrm{NH}_{4}{ }^{+}$sugerem que a $\mathrm{NH}_{3}$ é oriunda de fontes de emissões distantes, indicando que os efeitos da emissão poderão ter impactos em escala regional ao invés de apenas local. 
MENDES, L.B. et al. Os caminhos na natureza da amônia oriunda da produção animal: Parte I fontes, transformações e sumidouros. PUBVET, Londrina, V. 6, N. 24, Ed. 211, Art. 1406, 2012.

A intensidade em que cada um dos processos de deposição de $\mathrm{NH}_{3}$ e $\mathrm{NH}_{4}{ }^{+}$ na atmosfera (deposição a seco ou úmida), dependerá das características da região de deposição como tipos de clima e superfícies de deposição (vegetação, solo, superfícies aquosas, etc). Do ponto de vista da pesquisa científica, o monitoramento das deposições úmidas é mais simples enquanto que ainda não existe um método padrão para o monitoramento da deposição a seco.

As complexidades envolvidas no monitoramento da trajetória e transformações na natureza de uma pluma de $\mathrm{NH}_{3}$ emitida por instalação animal tornam este tema um desafio para o estado atual da pesquisa científica, e sugerem que este é um campo extremamente fértil para a exploração de novas técnicas de quantificação, desenvolvimento de modelos e potencialmente no estabelecimento de novos métodos de monitoramento.

\section{Referências}

Andersen, H. V.; Hovmand, M. F. 1999. Review of dry deposition measurements of ammonia and nitric acid to forest. Forest Ecology and Management 114 (1999) 5-18.

Baptista, F. J.; Bailey, B. J.; Randall, J. M.; Meneses, J. F. 1999. Greenhouse ventilation rate: Theory and measurement with tracer gas techniques. J. Agric. Engng. Res. (1999) 72, 363374.

Barbosa, L. C. A. 2000. Química orgânica: uma introdução para as ciências agrárias e biológicas. Ed. UFV.

Battye, R., Battye, W., Overcash, C., Fudge, S., 1994. Development and Selection of Ammonia Emission Factors. 68-D3-0034. US Environmental Protection Agency, Washington, DC.

Bouwman, A.F.; Lee, D.S.; Asman, W.A.H.; Dentener, F.J.; Van Der Hoek, K.W.; Olivier, J.G.J. 1997. A global high-resolution emission inventory for ammonia. Global Biogeochemical Cycles $11,561-587$.

Crutzen, J., 1983. Atmospheric interactions - homogeneous gas reactions of $\mathrm{C}, \mathrm{N}$ and $\mathrm{S}$ containing compounds. In: Bolin, B., Cook, R.B. (Eds.), The Major Biochemical Cycles and their Interactions. Scope 21, 67-114.

Dentener, F.J., Crutzen, P.J., 1994. A three-dimensional model of the global ammonia cycle. J. Atmos. Chem. 19, 331-369.

Erisman, J.W., Draaijers, G.P.J., Mennen, M.G., Hogenkamp, J.E.M., van Putten, E., Uiterwijk, W., Kemkers, E., Wiese, H., Duyzer, J.H., Otjes, R., Wyers, G.P., 1996. Towards development 
of a deposition monitoring network for air pollution of Europe; Deposition monitoring over the Speulder forest. Report no. 722108014, RIVM, Bilthoven, The Netherlands.

Finlayson-Pitts, B.J.; Pitts Jr., J.N. 1986. Atmospheric Chemistry: Fundamentals and Experimental Techniques. Wiley, New York.

Fowler, D.; Sutton, M.; Flechard, C.; Pitcairn, C. 1997. Ammonia sources, land-atmosphere exchange and efects: a European perspective. In: Proceedings of the Workshop on Atmospheric Nitrogen Compounds: Emissions, Transport, Transformation, Deposition and Assessment. North Carolina State University, Raleigh, NC, USA, pp. 36-46.

Grimm, E. 2009. Environmental Legistation in the European Union to Reduce Emissions from Livestock Production. Disponível em format pdf no site:

http://agrienvarchive.ca/bioenergy/download/grimm.pdf, acessado em 08/09/2011.

Groot Koerkamp, P. W. G.; Metz, J. H. M.; Uenk, G. H.; Philips, V. R.; Holden, M. R.; Sneath, R. W.; Shorf, J. L.; White, R. P.; Harting, J.; Seedorf, J.; Schroder, M.; Linkert, K. H.; Pedersen, S.; Takai, H.; Johnen, J. O.; Wathes, C. M. 1998. Concentrations and emissions of ammonia in livestock buildings in northern Europe. J. Agric. Engng. Res. (1998) 70, 79-95.

Hall, A.D., Miller, N.H.J., 1911. On the absorption of ammonia from the atmosphere. J. Agric. Sci. 4, 56-68.

Hartung J.; Phillips V.R. 1994. Journal of Agricultural Engineering Research, Vol.57 Issue 3,Pages 173-189.

Hutchings, N.J., Sommer, S.G., Andersen, J.M., Asman, W.A.H., 2001. A detailed ammonia emission inventory for Denmark. Atmospheric Environment 35, 1959-1968.

Junge, C. E.; Gustafson, P. E. 1957. On the distribution of seasalt over the United States and its removal by precipitation, Tellus $9,164-173$.

Krupa, S. V. 2003. Effects of atmospheric ammonia (NH3) on terrestrial vegetation: a review. Environmental Pollution 124 (2003), p.179-221.

Lee, J. D. 2009. Consise inorganic chemistry. Chapmann \& Hall, New York, U.S.A. 415 p.

Liu, Z.; Wang, L. 2006. Beasley, D. B. A review of Emission Models of Ammonia Released from Broiler Houses. ASABE Technical Paper 06-4101, St. Portland, OR: ASABE.

Medeiros, R.; Santos, B. J. M.; Freitas, M.; Silva, O. A.; Alves, F. F.; Ferreira, E. 2008. A adição de diferentes produtos químicos e o efeito da umidade na volatilização de amônia em cama de frango. Ciência Rural, v.38, p.2321-2326.

Moumen, N.; Yi, S. M.; Raymond, H. A.; Han, Y. J.; Holsen, T. 2004. Quantifying the dry deposition of ammonia in ammonia-rich and ammonia-poor environments using a surrogate surface approach. Atmospheric Environment. 38 (2004) 2677-2686.

Nääs, I. S.; Miragliotta, M. Y.; Baracho, M. S.; Moura, D. J. 2007. Ambiência em área de alojamento de frangos de corte: poeira e gases. Eng. Agrícola, v.27, n.2, p.326-335.

Nelson, D. L.; Cox, M. M. 2011. Lehninger: principles of biochemistry, 5th edition, W. H. Freeman and Company, New York, U. S. A. 1016. 
Owada, A. N.; Nääs, I. A.; Moura, D. J.; Baracho, M. S. 2007. Estimativa do bem-estar de frango de corte em função da concentração de amônia e grau de luminosidade no galpão de produção. Eng. Agrícola, v. 27, n.3, p.611-618.

Pain, B.F., van der Weerden, T.J., Chambers, B.J., Phillips, V.R., Jarvis, S.C. 1998. A new inventory for ammonia emissions from UK agriculture. Atmospheric Environment 32 (3), 309-313.

Saraz, J. A. O. 2010. Determinação experimental e modelagem em CFD das taxas de emissões de amônia de camas de aviários e distribuições de concentrações, temperatura e velocidade do ar no interior de galpões avícolas. Viçosa: $122 \mathrm{f}$. Tese (Doutorado em Construções Rurais). Departamento de Engenharia Agrícola, Universidade Federal de Viçosa, Viçosa, MG.

Shen, J.; Liu, X.; Zhang, Y.; Fangmeier, A.; Goulding, K.; Zhang, F. 2011. Atmospheric ammonia and particulate ammonium from agricultural sources in the North China Plain. Atmospheric Environment 45(2011), p.5033-5041.

Slack, A. V.; James, G. R. 1973. Ammonia: fertilizer science and technology series. Volume I. Marcel Dekker, Inc. New York.

Souza, P. A.; Melo, W. Z. 2011. Estimativa das emisões de amônia por excreta de animais domésticos para o estado do Rio de Janeiro. In: II Simpósio Internacional sobre Gerenciamento de Resíduos Agropecuários e Agroindustriais - II SIGERA, Foz do Iguaçu, Brasil.

Sutton, M. A.; Erisman, J. W.; Dentener, F.; Möller, D. 2008. Ammonia in the encvironment: From ancient times to the present. A review. Environmental Pollutionn 156 (2008) 583-604.

Tsitouridou, R.; Anatolaki, C. 2007. Atmospheric deposition of nitrogen, sulfur and chloride in Thessaloniki, Greece. Atmospheric Research 85 (2008) 413-428.

Twomey, S., 1977. Atmospheric Aerosols. Elsevier, Amsterdam, 302pp.

USDA, United States Department of Agriculture:

http://www.fas.usda.gov/psdonline/circulars/livestock_poultry.pdf, acessado em 15/09/2011.

Vigoderis, R. B.; Cordeiro, M. B. Tinôco, I. F. F.; Menegali, I.; Souza Junior, J. P.; Holanda, M. C. R. Avaliação do uso de ventilação mínima em galpões avícolas e sua influência no desempenho de aves de corte no período de inverno. R. Bras. Zootec., v.39, n.6, p.13811386.

Walker, J. T.; Aneja, V. P.; Cickey, D. A. 2001. Atmospheric transport and wet deposition of ammonium in North Carolina. Atmospheric Environment 34 (2000) 3407-3418.

Walker, J.T.; Whitall, D.R.; Robarge, W.; Paerl, H.W. 2004. Ambient ammonia and ammonium aerosol across a region of variable ammonia emission density. Atmospheric Environment 38, 1235-1246.

Warneck, P., 1988. Chemistry of the Natural Atmosphere, Academic Press, San Diego, 757pp.

Wesley, M.L.; Hicks, B.B. 1977. Some factors that affect the deposition rates of sulphur dioxide and similar gases to vegetation. J. Air Pollut. Control Ass. 27, 1110-1116. 